\title{
Relevance of the Antenatal Corticosteroids-to- Delivery Interval in the Prevention of Neonatal Respiratory Distress Syndrome Through the Eyes of Causal Inference: A Review and Target Trial
}

\section{Isabelle Dehaene ( $\boldsymbol{Q}$ isabelle.dehaene@ugent.be )}

Ghent University Hospital https://orcid.org/0000-0002-4826-6946

\section{Kristien Roelens}

Ghent University hospital

Koenraad Smets

Ghent University hospital

Johan Decruyenaere

Ghent University hospital https://orcid.org/0000-0002-3655-4318

\section{Research Article}

Keywords: Antenatal corticosteroids-to-birth interval, preterm birth, respiratory distress syndrome, causal inference, target trial

Posted Date: May 27th, 2021

DOI: https://doi.org/10.21203/rs.3.rs-477385/v1

License: (9) This work is licensed under a Creative Commons Attribution 4.0 International License.

Read Full License

Version of Record: A version of this preprint was published at Archives of Gynecology and Obstetrics on August 30th, 2021. See the published version at https://doi.org/10.1007/s00404-021-06213-5. 


\section{Abstract}

Purpose - To critically analyse the literature on the antenatal corticosteroids (ACS)-to-birth interval from a causal point of view and to present a solution to the problem of bias caused by post-hoc analysis.

Methods - Due to the post-hoc nature of the ACS-to-birth interval, a randomised controlled trial (RCT) of ACS versus placebo is not able to examine the importance of the interval. When an RCT is not feasible, for whatsoever reason, a target trial can be set up and an attempt can be made to answer the causal question of interest using observational data. An attempt was made to set up a target trial which could enable to examine the causal effect of the ACS-to-birth interval on neonatal outcomes. An analysis of current literature on the ACS-to-birth interval was done.

Results - The majority of studies aimed to examine the causal effect of the interval, but their study design only permitted to find associations between the interval and neonatal outcomes. Barriers for setting up a target trial are highlighted.

Conclusion - Evidence on the superiority of any ACS-to-birth interval is lacking and the question can only be addressed causally and become clinically relevant if baseline randomisation to ACS-to-birth intervals is made possible.

\section{Background}

Preterm birth (PTB), defined as delivery prior to 37 weeks' gestation, remains a challenging problem within the field of perinatology. In 1972, Liggins and Howie published a randomised controlled trial (RCT) on antenatal corticosteroids (ACS) for the prevention of respiratory distress syndrome (RDS) in preterm born infants. ${ }^{1}$ This trial was one of the most important trials in the history of perinatology. The introduction of ACS brought about a significant drop in neonatal morbidity and mortality. ${ }^{2}$ Based on a post-hoc analysis, the authors suggested that the greatest reduction of RDS incidence was seen in the group of children exposed between 24 hours and 7 days (24h-7d) before birth. ${ }^{1}$ Crowley et al. confirmed the importance of the $24 \mathrm{~h}-7 \mathrm{~d}$ interval in a secondary analysis of a meta-analysis on ACS. ${ }^{3}$ This presumed optimal interval influenced obstetrical management, which is reflected in, for instance, biomarker tests being designed to predict who will deliver within 7 days after presentation, or (weekly) repeat doses of ACS. ${ }^{4-9}$ In spite of that, it often occurs that women deliver term after having received ACS during an episode of threatened PTB or even prophylactically based on risk factors. ${ }^{10-13}$ There are concerns that these children are at risk for being small for gestational age and for developing neurodevelopment disorders. ${ }^{10,14-15}$ It is to be determined however if these outcomes are caused by ACS exposure or by the underlying etiology of preterm labour. ${ }^{14,16}$ Repeat courses of ACS are also a result of the presumed optimal interval. They are associated with an improved short-term neonatal outcome, which could support the presumed optimal $24 \mathrm{~h}-7 \mathrm{~d}$ interval. ${ }^{8,9}$ 
However, is this interval really the optimal interval? Is the decrease in neonatal morbidity higher when the ACS-to-birth interval is $24 \mathrm{~h}-7 \mathrm{~d}$ compared to other intervals?

This is not an easy question to answer. This is a question implying causality. To answer this type of question, one should perform an RCT. However, how can one randomise patients into an interval when the endpoint of the interval is unknown at the time of randomisation? This has resulted in a considerable amount of literature in which the ACS-to-birth interval is considered in post-hoc analyses of RCTs or in observational cohort studies. This article is a reflection on the reported literature on the ACS-to-birth interval, and an attempt to design a hypothetical trial examining the influence of the interval on neonatal outcome.

\section{Methods}

(1) A literature search on the relevance of the ACS-to-birth interval was performed in the PubMed database. The PICO and search string are available in S1.

(2) A risk of bias assessment was performed on the Liggins and Howie $\mathrm{RCT}^{1}$ using the Revised Cochrane risk-of-bias tool for randomised trials (RoB 2). ${ }^{17}$ This tool defines bias as "a systematic deviation from the effect of intervention that would have been observed in a large randomised trial without any flaws". We explored bias of their post-hoc analysis on the ACS-to-birth interval. The research question under consideration could be formulated as follows:

'Does the 24h-7d interval between ACS administration and birth cause a higher reduction in RDS compared to other ACS-to-birth intervals (<24h, 8-14d, >14d)?'.

(3) As concerns observational studies on the ACS-to-birth interval, we explored if the research question and methodology used had the intention to look for association or causation, if the results section was free of interpretation, and if discussion and conclusion were reported accordingly. ${ }^{18}$ We analysed if associational and causal language were used appropriately. Associational language was defined as expressions implying association without insinuating causation, for example 'is associated with', 'an association is observed', etc. Causal language was defined as expressions in which lie an assumption of causation, for example 'causes', 'changes', 'significantly reduced', 'explains', 'effect', 'attributes' etc. 'Confounding' is also a concept that only exists in causal studies.

(4) Following the approach of Hernán and Robins for enabling causal inference from observational studies, an attempt was made to formulate a target trial about the effect of the ACS-to-birth interval on the incidence of neonatal outcomes (for this exercise, RDS was chosen). ${ }^{19} \mathrm{~A}$ target trial is the description of a hypothetical randomised controlled trial that we would envisage to analyse the causal effect of a treatment. Causal inference techniques can then be used in an attempt to emulate the target trial with observational data. ${ }^{19,20}$ 
Hernán and Robins put a large emphasis on a well-described research question (a), described three key principles of causal inference (b), and described seven key components for adequately formulating a target trial (c). ${ }^{21}$

(a) Formulating the research question of the target trial

A causal research question requires an intervention. If we give treatment $A$, what is the effect on outcome $Y ?^{23}$

(b) The key principles of causal inference

Exchangeability

In randomised experiments the treated and untreated are exchangeable because the randomisation process ensures that independent predictors of the outcome are equally distributed between the treated and untreated groups. ${ }^{22}$ In an observational study, based on expert-knowledge, the researcher needs to define these independent predictors and foresee an equal distribution of these confounders between the treated and untreated group by applying particular statistical techniques, in which we will not go into detail (matching, stratification, standardisation, or inversed probability weighting). It is not possible to know if the researchers' assumption of which variables are confounders is correct, therefore there is always the risk of residual confounding.

\section{Consistency}

A treatment or intervention should be well defined. When a treatment is not well defined, multiple alternatives of the treatment are possible. Each treatment alternative can have another causal effect on the outcome and the average causal effect of the treatment will depend on the proportion of individuals who received each alternative. ${ }^{22}$ The treatment needs to be specified in detail.

Positivity

The probability of being assigned to each level of treatment should be greater than zero. ${ }^{22}$ Positivity is required for the confounding variables that are required for exchangeability:

$\operatorname{Pr}[A=a \mid L=\Lambda>0$ for all values $/$ with $\operatorname{Pr}[L=\Lambda \neq 0$ in the population of interest, with $A$ the treatment under consideration and $L$ the measured covariates considered to be confounders.

(c) Drafting the protocol

Seven key components of a target trial have been postulated. ${ }^{24}$ For this exercise we will consider RDS as the outcome.

1: eligibility. 
As for RCTs, eligibility criteria, defining who is included into or excluded from the trial, need to be formulated.

\section{2: treatment strategies}

Treatment should be assigned the moment at which eligibility criteria are met. And starting from then, outcomes need to be counted. These three elements (time when eligibility criteria are met, treatment is assigned, and study outcomes begin to be counted) need to be synchronised (time zero). If not, the target trial emulation can fail due to introduction of selection bias and immortal time bias. Immortal time bias can occur when treatment assignment precedes eligibility and patients die before being eligible. ${ }^{25}$

\section{3: assignment procedures}

To emulate the random assignment of strategies at baseline, we need to ensure exchangeability of the groups by adjusting for all confounders. ${ }^{24}$

\section{4: follow-upperiod}

The follow-up period needs to be defined.

\section{5: outcome}

Independent outcome validation might be warranted in observational research. ${ }^{24}$

\section{6: causal contrasts of interest}

The two common causal effects are the intention-to-treat and the per-protocol effect. The intention-totreat effect is the comparative effect of being assigned to a treatment strategy at baseline. The perprotocol effect is the comparative effect of patients who actually followed the treatment strategy and those who did not. ${ }^{24}$

\section{7: analysis plan}

An analysis plan would be an intention-to-treat or per-protocol analysis.

\section{Results}

(1) The literature search yielded 489 articles of which 21 articles were withheld (S2). Literature on the ACS-to-birth interval is summarised in Table S1. ${ }^{1,26-45}$

Two RCTs on ACS reported on the ACS-to-birth interval: the initial first $\mathrm{RCT}^{1}$, and the RCT of Schutte et al. ${ }^{26}$ Schutte et al performed a study (partially RCT (ACS vs placebo), partially observational (all women who received betamethasone)) to analyse the incidence of RDS in groups of preterm born children at different time intervals after admission of the mother. Although the goal was to investigate the relevance 
of time factors, the randomisation was for ACS or placebo. For the subgroup analysis of the time intervals, the number of patients in the time intervals was very small. Finally, the focus was less on the ACS-to-birth interval, but mainly on the admission-to-birth interval. ${ }^{26}$

All remaining 19 studies that focused on the neonatal outcome according to the ACS-to-birth interval were observational (17 in retrospective ${ }^{27-41,44,45}$ and two in prospective cohorts ${ }^{42,43}$ ). Four studies ${ }^{27-30}$ postulated no superiority of a certain interval, three studies $35,38,42$ concluded that an interval of $0-7 \mathrm{~d}$ was superior compared to other intervals, and eight studies $31,34,36,37,41,43-45$ confirmed the optimal interval of one or 2 to 7 days. The remaining four studies ${ }^{32,33,39,40}$ suggested a different interval (Table S1).

(2) We assessed the risk of bias in the Liggins and Howie trial using the $R O B 2$ tool. ${ }^{1,17}$ In Table S2, a risk of bias assessment is available for the primary research question of the RCT (ACS vs placebo) and for the research question of interest for this article (ACS-to-birth interval). For both research questions, the risk of bias was considered to be high. The same conclusion accounts for the study of Schutte et al. ${ }^{26}$

(3) Observational studies on the ACS-to-birth interval were prone to causal conclusions based on associational research questions and methodologies. A summary is provided in Table S3. ${ }^{27-45}$ None of the observational studies used causal inference techniques, however $89.5 \%$ used causal language. In $52.6 \%$ of articles, multiple regression was done to account for confounding. However, when considering an association, there is no need to correct for confounders. ${ }^{22}$ In our opinion, Peaceman et al. and Fuller et al. were the only ones who did not use any terms that implied causality. ${ }^{31,40}$

(4) We did an attempt to address the following research question in a hypothetical trial: 'Does the $24 h-7 d$ interval between ACS administration and birth cause a higher reduction in RDS compared to other ACS-tobirth intervals ( $<24 h, 8-14 d,>14 d)$ ?'

\section{(a) Research question}

An interval is not an intervention. It is an observation. An intervention would be to give ACS or not, or to repeat ACS or not. To transform the ACS-to-birth interval into an intervention, patients could be randomised to one of the four groups and labour could be induced so that birth occurs within one of the four intervals (change the endpoint of the interval). Of course, inducing labour to ensure one delivers preterm within a certain interval, would be unethical. An alternative would be to postpone ACS administration (change the start point of the interval). However, omitting ACS administration based on false negative predictions for birth within a certain interval would again be unethical. So, the research question cannot be answered, at least not if the actual, a posteriori known, interval is used. A possible solution would be to use the predicted interval at the time of ACS administration (see Key component 6 and 7).

(b) The 3 key principles of causal inference applied to the ACS-to-birth interval

Exchangeability 
To achieve (conditional) exchangeability between the patients in the four ACS-to-birth intervals considered, confounders need to be explored. Confounders are shared causes of treatment and outcome. A directed acyclic graph is a way to present these confounders, an example is presented in Figure 1.

\section{Consistency}

Different regimens of ACS are possible and different intervals can be considered. One regimen needs to be chosen for the purpose of the target trial. For example: two intramuscular injections of $12 \mathrm{mg}$ betamethasone (Celestone Chronodoseò, betamethasone acetate $(3 \mathrm{mg} / 1 \mathrm{~mL})$ and phosphate $(4$ $\mathrm{mg} / 1 \mathrm{~mL})$ ) with a $24 \mathrm{~h}$ interval. The ACS-to-birth intervals taken into consideration could be: $<24 \mathrm{~h}, 24 \mathrm{~h}-7 \mathrm{~d}$, $8-14 d$, and $>14 d$.

Positivity

There should be patients in each predefined ACS-to-birth interval.

(c) Target trial protocol

1: eligibility

Eligibility criteria could be:

1. All women at risk of spontaneous or iatrogenic PTB.

2. Presenting at a gestational age of 23 weeks and 5 days till 33 weeks and 5 days.

Exclusion criteria: Antenatally detected fetal congenital malformations with an effect on neonatal outcome and intra-uterine fetal death at presentation.

\section{2: treatment strategies}

The use of the ACS-to-birth interval as intervention is problematic and makes it impossible to establish a time zero. Birth falls after the baseline of the study and therefore is not known at the start of the study. Immortal time bias could arise when fetal death during the ACS-to-birth interval occurs.

Another issue is the possibility of repeating ACS. A large proportion of patients with the diagnosis of preterm labour does not give birth immediately (sometimes not even preterm). When experiencing a new episode of threatened PTB, a repeat course of ACS could be given. This further complicates the set-up of a target trial since this requires the availability of confounders for each administration of ACS (which might not all be available in the data) and the use of g-methods for time-varying-treatments.

\section{3: assignment procedures}

We cannot randomly assign patients to an interval, since we are interested in an 'intervention' only known post-randomisation. 


\section{4: $\underline{\text { follow-upperiod }}$}

The follow-up period of the mothers is until delivery. The follow-up of the neonates is until discharge from the neonatal unit. Criteria for discharge need to be set out. Neonates can be transferred back to the referring center and complications might still occur there. However, a diagnosis of RDS is very unlikely to be made after discharge of the neonatal intensive care unit.

\section{5: outcome}

When the neonatologist knows a mother has received ACS, he might be less likely to diagnose RDS. We think neonatologists rather focus on whether ACS were given and not within which interval birth occured. Therefore, we do not expect a problem with outcome validity for RDS. A sound, uniform definition of RDS, however, is mandatory.

\section{6: causal contrasts of interest}

If we would be able to know the ACS-to-birth interval (by being able to predict when birth will occur (if only we could!) or by ensuring that a patient delivers within the interval she is randomly assigned to (unethical)), we would be able to assign patients to one of the four intervals. If, however, the patients delivered earlier or later than expected, they would belong to another interval. The intention-to-treat effect would compare the outcome of patients assigned to a certain interval, the per-protocol effect would compare the outcome of patients who delivered in a certain interval.

\section{7: analysis plan}

In the intention-to-treat analysis, we would explore the effect of the predicted ACS-to-birth interval on RDS. We can also do a per-protocol analysis by looking at the effect of the ACS-to-birth interval in which the patient actually delivered on RDS. For this, we not only need to adjust for confounding at baseline, but also for post-randomisation confounding. Post-baseline prognostic factors need to be identified and adjusted for. These post-baseline prognostic factors might not always be readily available in the data, which will lead to residual confounding.

\section{Comments}

The research question of interest cannot be formulated causally based on observed ACS-to-birth intervals. It is impossible to set a time zero, a time at which eligibility, assignment to intervention and start of follow-up coincide. Only associational studies and conclusions are possible, exploring an association between the different intervals and the neonatal outcome. Children born at a certain interval can have different characteristics than children born at other intervals. This was also addressed by Gates and Brocklehurst, who discussed that the evidence on the relevance of the ACS-to-birth interval is based on unsound subgroup analyses. ${ }^{46}$ 
Conducting multiple tests is associated with a risk of obtaining significant (false positive) results due to chance alone (familywise error rate). ${ }^{36}$ When subgroups are not specified before doing an RCT, the risk of the analyses being a result of looking for significant differences between subgroups with the goal of finding any significant result, is high. One should always remain vigilant about post-hoc and subgroup analyses, and interpret them with great caution. Subgroup effects gain credibility when the number of subgroups examined is small, the subgroups are prespecified, the treatment effect between the groups is considerably high, the sample sizes are large, and the observed effect is clinically plausible. Preferably, the effects are consistent and duplicated in other studies. ${ }^{37}$ The outcome of the Liggins RCT was considerably biased due to subdivision of the study population post-randomisation in etiologic groups of PTB. ${ }^{1}$ By doing this, the authors introduced a high risk of hampering the exchangeability between the intervention groups per etiologic group, which eliminated the advantages of doing an RCT. Moreover, by comparing multiple groups, they created smaller sample sizes, and introduced a risk of inflated familywise error rates. ${ }^{36}$ As concerns the interval, the significant difference in RDS incidence in the $24 \mathrm{~h}$ $7 \mathrm{~d}$ group seems most likely to be due to chance. The groups compared were small ( 28 neonates in the treatment group, 24 in the control group), and the probability of a type I error was large (p-value of .03 without multiple testing correction, when performing Bonferroni correction, the $\mathrm{p}$-value reducing the risk of a type I error, should have been .0125). ${ }^{1}$ Finally, the subgroup analysis was not prespecified and concerned a post-randomisation event. Hirji and Fagerland described four concerns regarding outcome based subgroup analysis: reduced power, overdone post-hoc analysis, selective reporting, and overinterpretation. The subgroup analysis of the ACS-to-birth interval in the Liggins trial is prone to all these concerns due to respectively a small subset, a not prespecified analysis, highlighting of significant findings, and an overt overinterpretation of the result of the subgroup analysis which is reflected in the importance the interval gained in obstetrical literature and practice. ${ }^{49}$ In general, whenever subgroups are considered in RCTs, there is a risk of post-randomisation confounding due to the risk of lack of exchangeability between the treatment and non-treatment arm within the subgroup. ${ }^{50}$

The cohort studies are also marked by a high risk of selection bias. Cohort follow-up did not start when ACS were administered, but study participants were selected based on gestational age or weight at birth. Follow-up of women who delivered at term is absent. The risk of collider-stratification bias is high and even more complex than its pure form, since the collider is partially part of the 'intervention' (Figure 1). ${ }^{51,52}$ Controlling for confounders in non-randomised studies in order to explore causality, requires an insight in which variable is a confounder. When using a DAG, designed based on subject-matter knowledge, confounders are shared causes of exposure and outcome. Once confounders are identified, they can be used in a propensity score model, often a multivariate logistic regression model, which will allow exchangeability of the treatment groups. When the principles of positivity and consistency are not violated, one can now use causal inference methods to explore if the exposure has a (causal) effect on the outcome. Interestingly, even the design of a DAG is challenging when considering the interval. An alternative to Figure 1 is adding the interval as a node, which has implications on which variables are considered to be confounders. Alternatively, variables can also be included in a multivariate logistic 
regression model to enable outcome prediction, without aiming to explore a causal relationship between the exposure/predictor and the outcome.

\section{Conclusions}

In our opinion, we do not know if the ACS-to-birth interval has an influence on outcomes of preterm born children. An RCT comparing ACS with placebo with a considerably large sample size and prespecified subgroup analysis of the interval, correcting for multiple testing, could shed more light on the importance of the interval. However, due to the widespread use of ACS, and for financial and ethical reasons, such a trial is unlikely to happen. Moreover, the utility of the ACS-to-birth interval in daily practice is questionable, since the date of birth is not known at the moment of ACS administration.

The main problem with the ACS-to-birth interval is that the end-point of the interval, birth, is an event in the future of which the time of occurrence is unknown at the moment the patient presents with threatening preterm labour, making it impossible to translate the interval to an intervention which would allow formulating a well-defined research question and selecting an appropriate study design. A 'simple' RCT or cohort study is not able to tackle this problem. More advanced techniques are mandatory, if ever it is possible to disentangle this web. If we would have an accurate prediction tool for time of delivery after ACS administration, people could be randomised to predicted ACS-to-birth intervals. In the awaiting of such a tool, an intermediate approach could be to investigate the risk of (for example) RDS according to the probability of birth at a certain time point, starting from the time of ACS administration. Another possibility would be to work with dynamic treatment strategies. Observational data and causal inference techniques can be used for this approach and risk of bias can be assessed with the Risk Of Bias In Nonrandomised Studies of Interventions tool, which allows for risk of bias assessment of non-randomised studies of interventions. ${ }^{53}$ We plan to do this "disentangling" analysis and invite research groups with data concerning the ACS-to-birth interval to join us.

\section{Declarations}

\section{Contribution of authorship}

ID: Conceptualization, Writing - Original Draft, Writing - Review \& Editing. KR: Writing - Review \& Editing, Supervision. KS: Writing - Review \& Editing, Supervision, JD: Writing - Review \& Editing, Supervision.

\section{Acknowledgments}

This article is part of the PhD project of Isabelle Dehaene, called "PREdiction tool for preTerm laboUr and Neonatal outcome" (PRETURN). We would like to thank all other researchers involved in PRETURN, in particular Johan Steen, statistician at Ghent University and Ghent University Hospital, Belgium. He is a specialist in causal inference with a special interest in time-varying treatments. This work would not have been possible without his input. 
Funding: Isabelle Dehaene is funded by a scholarship of FWO (1700520N). The funding body played no role in the creation of this manuscript.

Conflicts of interest: the authors declare that they have no conflict of interest

Availability of data and material: not applicable

Code availability: not applicable

Ethics approval: not applicable

Consent to participate / for publication: not applicable

\section{References}

1. Liggins GC, Howie RN (1972) A controlled trial of antepartum glucocorticoid treatment for prevention of the respiratory distress syndrome in premature infants. Pediatrics 50(4):515-525

2. WHO Recommendations on Interventions to Improve Preterm Birth Outcomes. Available at https://apps.who.int/iris/bitstream/handle/10665/183037/9789241508988_eng.pd?sequence=1 Retrieved February 4th 2019

3. Crowley P (1995) Antenatal corticosteroid therapy: a meta-analysis of the randomized trials, 1972 to 1994. Am J Obstet Gynecol 173(1):322-335

4. Crowther C, McKinlay C, Middleton P, Harding J. Repeat doses of prenatal corticosteroids for women at risk of preterm birth for improving neonatal health outcomes. The Cochrane database of systematic reviews 2015;(7). Art. No.: CD003935

5. Bruijn M, Vis J, Wilms F, Oudijk M, Kwee A, Porath M et al (2016) Comparison of the Actim Partus test and the fetal fibronectin test in the prediction of spontaneous preterm birth in symptomatic women undergoing cervical length measurement. Eur J Obstet Gynecol Reprod Biol 206:220-224

6. Watson H, Carter J, Seed P, Tribe R, Shennan A (2017) The QuiPP App: a safe alternative to a treat-all strategy for threatened preterm labor. Ultrasound Obstet Gynecol 50(3):342-336

7. Demirci O, Ünal A, Demirci E, Sözen H, Akdemir Y, Boybek E et al (2011) Sonographic measurement of cervical length and risk of preterm birth. The Journal of Obstetrics Gynaecology Research 37(7):809-814

8. Crowther C, Aghajafari F, Askie L, Asztalos E, Brocklehurst P, Bubner T et al (2012) Repeat prenatal corticosteroid prior to preterm birth: a systematic review and individual participant data metaanalysis for the PRECISE study group (prenatal repeat corticosteroid international IPD study group: assessing the effects ousing the best level of evidence) - study protocol. Syst Rev 1:12

9. Crowther C, Middleton P, Voysey M, Askie L, Zhang S, Martlow T et al (2019) Effects of repeat prenatal corticosteroids given to women at risk of preterm birth: An individual participant data metaanalysis. PLoS Med 16(4):e1002771 
10. Melamed N, Asztalos E, Murphy K, Zaltz A, Redelmeier D, Shah B, Barret J (2019)

Neurodevelopmental disorders among term infants exposed to antenatal corticosteroids during pregnancy: a population-based study. BMJ Open 9:e031197. doi:10.1136/bmjopen-2019-031197

11. Parnell L, Ayuk P (2020) Prophylactic antenatal corticosteroids for fetal lung maturity: Known unknowns and unknown unknowns. Case Rep Womens Health 28:e00242. doi:10.1016/j.crwh.2020.e00242

12. Murphy D, Caukwell S, Joels L, Wardle P (2002) Cohort study of the neonatal outcome of twin pregnancies that were treated with prophylactic or rescue antenatal corticosteroids. AJOG 187(2):483-488

13. Jobe A, Goldenberg G (2018) Antenatal corticosteroids: an assessment of anticipated benefits and potential risks. AJOG 219(1):62-74

14. Espinoza $\mathrm{J}$ et al (2007) An episode of preterm labor is a risk factor for the birth of a small-forgestational-age neonate. AJOG 196:574 :.e1-574.e6

15. Paules $\mathrm{C}$ et al (2017) Threatened preterm labor is a risk factor for impaired cognitive development in early childhood. AJOG 216:157.e1-157.e7

16. Romero R et al (2017) Is an episode of suspected preterm labor that subsequently leads to a term delivery benign? AJOG 216:89-94

17. Sterne J, Savović J, Page M, Elbers R, Blencowe N, Boutron I et al (2019) RoB 2: a revised tool for assessing risk of bias in randomised trials. BMJ: Britisch Medical Journal 366:14898

18. Hernán M (2018) The C-Word: scientific euphemisms do not improve causal inference from observational data. Am J Public Health 108:616-619

19. Hernán M, Robins J (2016) Using big data to emulate a target trial when a randomized trial is not available. Am J Epidemiol 183(8):758-764

20. Prosperi M, Guo Y, Sperrin M, Koopman J, Min J, He X et al (2020) Causal inference and counterfactual prediction in machine learning for actionable healthcare. Nat Mach Intell 2:369-375

21. Hernán M, Robins J (2016) Using big data to emulate a target trial when a randomized trial is not available. Am J Epidemiol 183(8):758-764

22. Hernán M, Robins J. Causal inference. What if. CRC Press Taylor \& Francis Group A Chapman \& Hall book; 2020. Available at https://cdn1.sph.harvard.edu/wp-

content/uploads/sites/1268/2019/02/hernanrobins_v1.10.38.pdf. Retrieved March 30th 2020

23. Hernán M, Taubman S (2008) Does obesity shorten life? The importance of well-defined interventions to answer causal questions. Internation Journal of Obesity: journal of the International Association for the Study of Obesity 32:S8-S14

24. Hernán M, Robins J (2016) Using big data to emulate a target trial when a randomized trial is not available. Am J Epidemiol 183(8):758-764

25. Hernán M, Sauer B, Hernandez-Dias S, Platt R, Shrier I (2016) Specifying a target trial prevents immortal time bias and other self-inflicted injuries in observational analyses. J Clin Epidemiol 79:70- 
26. Schutte M, Treffers P, Koppe J (1983) Threatened preterm labor: the influence of time factors on the incidence of respiratory distress syndrome. Obstetrics Gynecology 146(4):395-400

27. McNamara M, Bottoms S (1998) The incidence of respiratory distress syndrome does not increase when preterm delivery occurs greater than 7 days after steroid administration. The Australian New Zealand Journal of Obstetrics Gynaecology 38(1):8-10

28. Vemillion S, Soper D, Newman R (2001) Is betamethasone effective longer than 7 days after treatment? Obstetrics Gynecology 97(4):491-493

29. Smrcek J, Schwarau N, Kohl M, Berg C, Geipel A, Krapp M et al (2005) Antenatal corticosteroid therapy in premature infants. Arch Gynecol Obstet 271:26-32

30. Sehdev H, Abbasi S, Robertson P, Fischer L, Marchiano D, Gerdes J et al (2004) The effects of the time interval from antenatal corticosteroid exposure to delivery on neonatal outcome of very low birth weight infants. Am J Obstet Gynecol 191:1409-1413

31. Peaceman A, Bajaj K, Kumar P, Grobman W (2005) The interval between a single course of antenatal steroids and delivery and its association with neonatal outcomes. Am J Obstet Gynecol 193:11651169

32. Ring A, Garland J, Stafeil B, Carr M, Peckman G, Pircon R. The effect of a prolonged time between antenatal corticosteroid administration and delivery on outcomes in preterm neonates: a cohort study. American Journal of Obstetrics and Gynecology 2007;196:457.e\&-457.e6

33. Ferguson S, Allen V, Craig C, Allen A, Dodds L (2009) Timing of indicated delivery after antenatal steroids in preterm pregnancies with severe hypertension. Hypertension in Pregnancy 28:63-75

34. Waters T, Mercer B (2009) Impact of timing of antenatal corticosteroid exposure on neonatal outcomes. Journal of Maternal Fetal Neonatal Medicine 22(4):311-314

35. Wilms F, Vis Y, Pattinaja D, Duin R, Stam M, Reuvers J et al (2011) Relationship between the time interval from antenatal corticosteroid administration until preterm birth and the occurence of respiratory morbidiy. Am J Obstet Gynecol 205:e1-e7

36. Kuk J-Y, A J-J, Cha H-H, Choi S-J, Vargas J, Oh S-y et al (2013) Optimal time interval between a single course of antenatal corticosteroids and delivery for reduction of respiratory distress syndrome in preterm twins. Am J Obstet Gynecol 209:256.e1-256.e7

37. Melamed N, Shah J, Soraisham A, Yoon E, Lee S, Shah P et al (2015) Association between antenatal corticosteroid administration-to-birth interval and outcomes of preterm neonates. Obstetrics Gynecology 125:1377-1384

38. Kosinska-Kazzynska K, Szymusik I, Urban P, Zachara M, Wielgos M (2016) Relation between time interval from antenatal corticosteroids administration to delivery and neonatal outcomes in twins. The Journal of Obstetrics Gynaecology Research 42(6):625-631

39. Liebowitz M, Clyman R (2016) Antenatal betamethasone: a prolonged time interval from administration to delivery is associated with an increased incidence of severe intraventricular hemorrhage in infants born before 28 weeks gestation. The Journal of Pediatrics 177:114-120 
40. Fuller K, DeGroff S, Borgida A (2017) Neonatal outcomes based on antenatal corticosteroid exposure time for infants delivered between 23 and 34 weeks' gestation. Clin Exp Obstet Gynecol 44(2):252258

41. Yasuhi I, Myoga M, Suga S, Sugimi S, Umezaki Y, Fukuda M et al (2017) Influence of the interval between antenatal corticosteroid therapy and delivery on respiratory distress syndrome. The Journal of Obstetrics Gynaecology Research 43(3):486-491

42. Norman M, Piedvache A, Borch K, Drasbek Huusom L, Edstedt Bonamy A-K, Howell E et al (2017) Association of short antenatal corticosteroid administration-to-birth intervals with survival and morbidity among very preterm infants. JAMA Pediatrics 171(7):678-686

43. Norberg H, Kowalski J, Marsal K, Norman M (2017) Timing of antenatal corticosteroid administration and survival in extremely preterm infants: a national population-based cohort study. Br J Obstet Gynaecol 124:1567-1574

44. Lau H, Tung J, Wong T, Tan P, Tagore S (2017) Timing of antenatal steroids exposure and its effects on neonates. Arch Gynecol Obstet 296:1091-1096

45. Frändberg J, Sandblom J, Bruschettini M, Marsal K, Kristensen K (2018) Antenatal corticosteroids: a retrospective cohort study on timing, indications and neonatal outcome. Acta Obstetrica et Gynecologica Scandinavica 97:591-597

46. Gates S, Brocklehurst $P$ (2007) Decline in effectiveness of antenatal corticosteroids with time to birth: real or artefact. BMJ 335:77-79

47. Field A, Miles J, Field Z (2012) Discovering statistics in R 2. SAGE Publications

48. Naggara O, Raymond J, Guilbert F, Altman D (2011) The problem of subgroup analyses: an example from a trial on ruptured intracranial aneurysms. American Journal of Neuroradiology 32:633-636

49. Hirji K, Fagerland M (2009) Outcome based subgroup analysis: a neglected concern. Trials 10:33. doi:10.1186/1745-6215-10-33

50. Desai M, Pieper K, Mahaffey K (2014) Challenges and solutions to pre- and post-randomization subgroup analyses. Curr Cardiol Rep 16:531

51. Snowden J, Basso O. Causal inference in studies in preterm babies: a simulation study BJOG 2017. DOI: 10.1111/1471-0528.14942

52. VanderWeele T, Mumford S, Schisterman E (2013) Conditioning on intermediates in perinatal epidemiology. Epidemiology 23(1):1-9

53. Sterne J, Hernán M, Reeves B, Savovic J et al (2016) ROBINS-l: a tool for assessing risk of bias in non-randomised studies of interventions. BMJ 355:i4949. DOI 10.1136/bmj.i4919

\section{Figures}




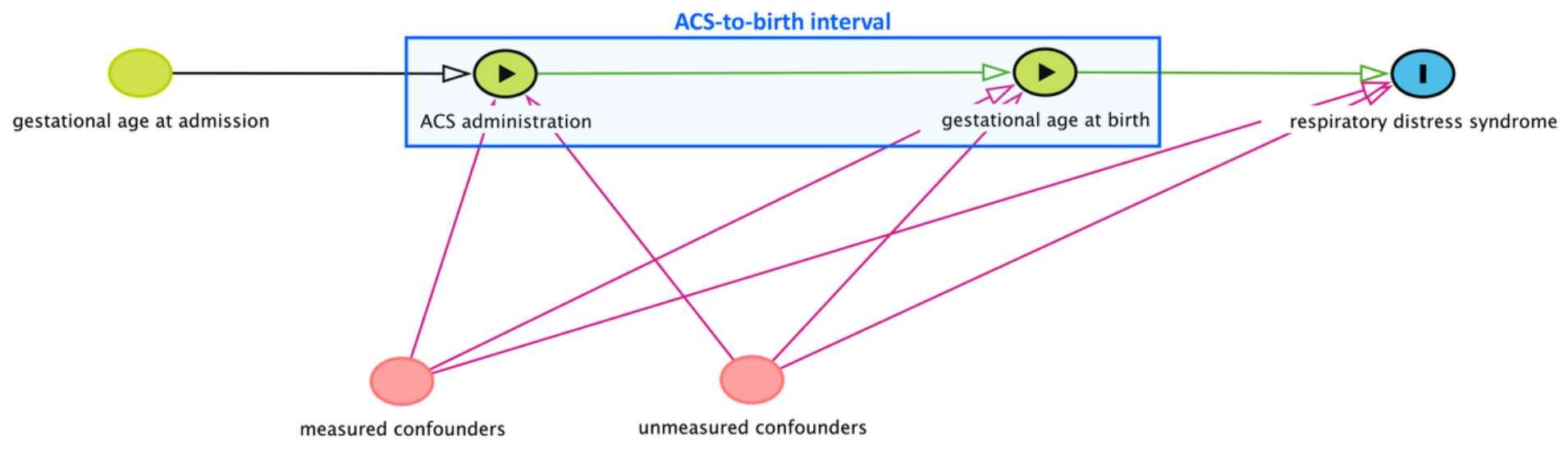

\section{Figure 1}

Directed Acyclic Graph depicting the relationship between the antenatal corticosteroids-to-birth interval and short-term neonatal outcome, for example respiratory distress syndrome. Abbreviations: PTB = preterm birth, GA = gestational age, ACS = antenatal corticosteroids, ART = artificial reproduction techniques, IUT = intra-uterine transfer, $\mathrm{n}=$ number of fetuses, $\mathrm{CRP}=\mathrm{C}$-reactive protein, $\mathrm{CL}=$ cervical length, IUGR = intra-uterine growth restriction, RDS = respiratory distress syndrome, $\mathrm{NICU}=$ neonatal intensive care unit

\section{Supplementary Files}

This is a list of supplementary files associated with this preprint. Click to download.

- PICO.docx

- PRISMA.docx

- Table1.docx

- Table2.docx

- Table3.docx 